\title{
A IMPORTÂNCIA DOS ESTUDOS DE PRÉ-FORMULAÇÃO NA ESTABILIDADE DOS PRODUTOS COSMÉTICOS
}

\section{THE IMPORTANCE OF PRE-FORMULATION IN THE STABILITY OF COSMETIC PRODUCTS}

\author{
Gizzelle Delfino Araújo Ladeira ${ }^{1}$ \\ Ana Sara Alvernaz Alves ${ }^{2}$ \\ Brenda dos Santos Mota ${ }^{3}$ \\ Jeice Aparecida Rosa ${ }^{4}$ \\ Maressa Gabrielle Rodrigues Gomes ${ }^{5}$ \\ Rafaela Coelho Tavares ${ }^{6}$ \\ Quéren Marques Lima Xavier ${ }^{7}$
}

RESUMO: Os produtos cosméticos podem sofrer danos, perder sua eficácia e segurança, o que justifica apresentar a importância da etapa de desenvolvimento, em destaque a fase de pré-formulação, na estabilidade física, química e microbiológica, desde o desenvolvimento até o cumprimento do prazo de validade estipulado pelo fabricante. O objetivo geral deste trabalho foi buscar informações que apresentassem a importância dos estudos de préformulação e confiabilidade dos cosméticos, abordando a complexa fase de desenvolvimento da formulação, a avaliação da estabilidade dos cosméticos, os fatores que influenciam na qualidade, os parâmetros avaliativos, os testes e os critérios para aprovação. Para tanto, este trabalho foi pautado em algumas revisões bibliográficas de forma qualitativa que tratam da temática, disponíveis em livros, tese, dissertações, monografias e artigos, priorizando trabalhos mais recentes e inovadores. A partir da revisão bibliográfica foi possível mostrar a necessidade da fase de pré-formulação bem delineada como etapa essencial para garantir produtos cosméticos estáveis, seguros e eficazes.

\footnotetext{
IDocente da Faculdade Única de Ipatinga/MG (FUNIP)/ Departamento de Farmácia. Mestrado em Ciências Farmacêuticas pela Universidade Federal de Minas Gerais. Especialização em Manipulação Magistral Alopática pelo Instituto Racine. Graduação em Farmácia, com Habilitação em Farmácia Industrial, pela Universidade Federal de Minas Gerais. E-mail: gizzaladeira@gmail.com

${ }^{2}$ Discente do curso de Farmácia da Faculdade Única de Ipatinga/MG (FUNIP). Email: anasaraalvernazfc@hotmail.com

${ }^{3}$ Discente do curso de Farmácia da Faculdade Única de Ipatinga/MG (FUNIP). Email: brenda_smota@hotmail.com

${ }^{4}$ Discente do curso de Farmácia da Faculdade Única de Ipatinga/MG (FUNIP). Email: jeiceaparecidarosa@hotmail.com

${ }^{5}$ Discente do curso de Farmácia da Faculdade Única de Ipatinga/MG (FUNIP). Email:maressathucar@gmail.com

6 Discente do curso de Farmácia da Faculdade Única de Ipatinga/MG (FUNIP). Email: rafaelacoelhot@gmail.com

${ }_{7}^{7}$ Discente do curso de Farmácia da Faculdade Única de Ipatinga/MG (FUNIP). Email: qm.xavier@hotmail.com
} 
Palavras-chave: Desenvolvimento. Cosméticos. Pré-formulação. Estabilidade.

ABSTRACT: Cosmetic products can suffer damage, lose their effectiveness and safety, which justifies the importance of the development stage, in special the pre-formulation phase, in physical, chemical and microbiological stability, since development to compliance with the validated deadline stipulated by the manufacturer. The general objective of this work was to seek information that showed the importance of preformulation studies in the stability and reliability of cosmetics, addressing a complex phase of base development, the evaluation of cosmetic stability, factors influencing stability, parameters of stability assessment, stability tests and approval criteria. Therefore, this work was based on some bibliographical reviews in a qualitative way that deal with the theme, available in books, thesis, dissertations, monographs and articles, prioritizing more recent and innovative works. Based on the literature review, it was possible to show the need for a well-designed pre-formulation phase as an essential step to ensure stable, safe and effective cosmetic products.

Keywords: Development. Cosmetics. Pre-formulation. Stability.

\section{INTRODUÇÃO}

A indústria de cosméticos tem se desenvolvido constantemente com produtos cada vez mais inovadores. Nos últimos anos, diferentes conceitos surgiram em cosméticos e os consumidores estão prestando cada vez atenção nos ingredientes que compõem os produtos que usam diariamente (REBELLO, 2019).

Os cosméticos são usados para o cuidado e o embelezamento do corpo. No passado, seus componentes eram extraídos principalmente de plantas, animais e minerais, mas, com o avanço da tecnologia, produtos químicos sintéticos foram adicionados às formulações desses produtos (CORNÉLIO; ALMEIDA, 2020).

Antes de serem liberados para o mercado, a qualidade dos cosméticos precisa ser avaliada e aprovada. A Agência Nacional de Vigilância Sanitária (ANVISA) publicou um Guia de Estabilidade de Produtos Cosméticos, que apresenta estudos e informações com o objetivo de garantir a manutenção das características dos produtos durante seu prazo de validade, orientando os profissionais do setor e avaliadores dos órgãos reguladores, com o intuito de ressaltar a importância da qualidade como critério para a proteção e promoção da saúde na população. (BRASIL, 2004).

A pré-formulação é a etapa no desenvolvimento de fármacos e formas farmacêuticas que precede a formulação em grande escala propriamente dita, na qual são avaliadas diversas características dos componentes do produto cosmético candidato e 
processo de fabricação (AULTON; TAYLOR, 20I6). Na prática, os produtos cosméticos são raramente associados com sérios danos ao consumidor, mas isto não significa que os produtos cosméticos sejam sempre seguros e estáveis, especialmente, se for considerar os efeitos à longo prazo. Partindo do pressuposto de que estes produtos podem sofrer danos ou perder sua eficácia, esse trabalho se justifica em mostrar a importância da etapa de desenvolvimento dos produtos cosméticos, a partir do estudo de estabilidade, a fim de garantir eficácia e segurança ao consumidor (CORNÉLIO; ALMEIDA, 2020).

O objetivo deste artigo foi buscar informações que apresentassem a importância dos estudos de pré-formulação e confiabilidade dos cosméticos, abordando a fase de desenvolvimento, destacando a etapa de pré-formulação, os fatores que influenciam, os parâmetros de avaliação, os testes e critérios para aprovação.

\section{METODOLOGIA}

A pesquisa foi baseada em uma revisão bibliográfica de forma qualitativa tendo como critério plataformas de base como Scielo, Pubmed e Google acadêmico, a partir de artigos, monografias, dissertações e livros que discorrem sobre o estudo da pré-formulação

e estabilidade dos produtos cosméticos, por intermédio do uso das palavras chaves: Desenvolvimento, Cosméticos, Pré-formulação, Estabilidade. Um levantamento de 40 artigos foi realizado e 26 artigos selecionados para a execução do trabalho. Foram utilizados estudos publicados de 2004 a 2021, priorizando os trabalhos mais recentes que corroborassem com a temática abordada, exceto os livros que variam de acordo com o ano de publicação. Os trabalhos que não abordaram as ideias principais propostas neste trabalho e que não se enquadraram nos critérios de inclusão foram excluídos.

\section{Desenvolvimento}

\section{I Produtos cosméticos}

De acordo com Cornélio e Almeida (2020) os produtos cosméticos são constituídos de formulações naturais ou sintéticas com diversos tipos de aplicação ao organismo humano. Estes produtos são desenvolvidos e utilizados para o cuidado da pele, unhas e cabelo, com o intuito de higienizar, proteger, perfumar, maquiar, corrigir e manter em bom estado o corpo humano (REBELLO, 2019). 
A Agência Nacional de Vigilância Sanitária (ANVISA) na RDC n. o7/2015 classifica os produtos de cosméticos de acordo com suas características de segurança em Grau i e Grau 2 conforme é apresentado na Tabela i a seguir, sendo os produtos de Grau I os produtos com risco mínimo e os produtos de Grau 2, produtos com risco potencial (BRASIL, 2015).

Tabela I - Classificação dos produtos

\begin{tabular}{|c|l|}
\hline Produtos Grau I & $\begin{array}{l}\text { São produtos que se caracterizam por possuírem propriedades básicas ou } \\
\text { elementares, cuja comprovação não seja inicialmente necessária e não requeiram } \\
\text { informações detalhadas quanto ao seu modo de usar e suas restrições de uso, devido } \\
\text { às características intrínsecas do produto. }\end{array}$ \\
\hline Produtos Grau 2 & $\begin{array}{l}\text { São produtos de higiene pessoal, cosméticos e perfumes cuja formulação cumpre } \\
\text { com a definição adotada na Resolução e que possuem indicaçóes específicas, cujas } \\
\text { características exigem comprovação de segurança e/ou eficácia, bem como } \\
\text { informações e cuidados, modo e restrições de uso. }\end{array}$ \\
\hline
\end{tabular}

Fonte: BRASIL, 2015.

O crescimento do mercado de cosméticos está relacionado às mudanças nas condições de consumo e ao aumento do interesse dos consumidores pelos tipos de produtos disponíveis (FURTADO; SAMPAIO, 2020). Isso aumenta o rigor do processo de pesquisa e desenvolvimento de cosméticos, pois os consumidores se tornam mais exigentes e procuram por produtos mais adequados às suas necessidades. Com isso, para se desenvolver um cosmético é preciso levar em consideração diversos fatores que afetam a qualidade e aceitação do produto, havendo necessidade de um criterioso processo de escolha de materiais e processos de produção (VOGEL et al., 2020).

\subsection{Desenvolvimento de produtos cosméticos}

Segundo Clepf, Martinelli e Campos (2015) o desenvolvimento de cosméticos é um processo complexo que inclui várias etapas para a criação de um produto adequadamente qualificado para o uso. Seu desenvolvimento se dá por meio de pesquisas que permitirão analisar as matérias primas, as técnicas de formulação e os novos conceitos, desde a ideia inicial até a concepção e comercialização do produto.

A primeira fase do desenvolvimento de um cosmético é gerar uma ideia promissora que irá direcionar para a elaboração do produto e o passo seguinte é a formulação, etapa 


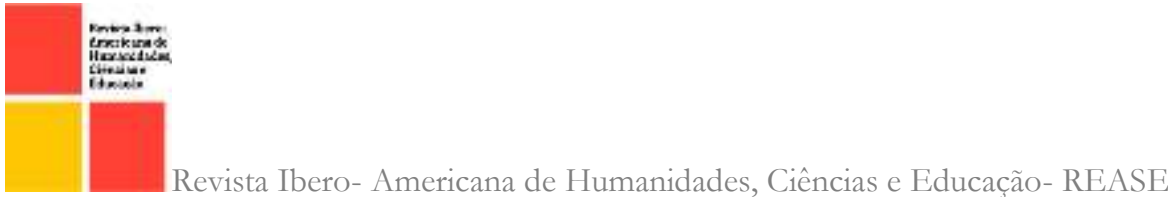

essencial para se compreender os mecanismos de ação compostos na fórmula e os processos envolvidos na fabricação do produto (COSTA et al., 2018).

Através da ideia inicial, elabora-se uma lista de matérias-primas que podem ou não ser utilizadas no desenvolvimento do cosmético. Por meio dessa lista realiza-se a preparação dos protótipos do produto para avaliar a estabilidade e funcionalidade da formulação. Em seguida, são realizados testes para avaliar a confiabilidade e aceitabilidade do protótipo. De maneira mais ampla, faz-se os testes de segurança, estabilidade, validade, eficácia e se necessários os testes clínicos, dentro das exigências para a regularização e registro do produto (CLEPF, MARTINELLI; CAMPOS, 2015; REBELLO, 2019; CORNÉLIO; ALMEIDA, 2020).

\subsubsection{A fase de pré formulação}

A pré-formulação é uma etapa do desenvolvimento de um produto projetada para otimizar o processo de transformação das matérias-primas da formulação em produtos farmacêuticos. Durante esse período as propriedades físicas, químicas e microbiológicas do produto serão analisadas e a qualidade desta etapa constitui um diferencial na criação de um cosmético. Nesta fase é essencial analisar os fatores que podem influenciar na vida útil do produto, assim como os procedimentos básicos a serem empregados para minimizar problemas com o produto acabado (PAIVA, 2016; AULTON; TAYLOR, 2016).

Para se propor uma formulação piloto, primeiramente apresenta-se qual o objetivo da elaboração do cosmético, considerando os diversos fatores envolvidos na escolha das matérias-primas, condições de processamento industrial, toxicidade e riscos ambientais, sendo a escolha das matérias-primas a parte mais importante do processo. Durante esta fase inicial pode ser previsto possíveis reações de hidrólise, oxidação, fotodegradação e incompatibilidade química entre os componentes da formulação, identificando mecanismos potenciais de futuros problemas de estabilidade (CLEPF, MARTINELLI; CAMPOS, 2015; SILVA, 2017).

De acordo com Silva (2017) o estudo de pré-formulação é essencial, pois a formulação de cosmético é de certo modo complexa e utilizam-se diferentes matériasprimas. As matérias-primas são geralmente classificadas como ingredientes ativos ou adjuvantes farmacotécnicos. O princípio ativo é uma substância química que promoverá a atividade terapêutica. Os excipientes são componentes que devem ser inertes, adicionados 
à formulação para proporcionar diferentes formas farmacêuticas e garantir a qualidade, minimizando os problemas relacionados à eficácia e segurança do produto (FERREIRA, 2016; PAIVA, 2016; VOGEL et al., 2020).

Cada substância apresenta características específicas que devem ser consideradas antes do desenvolvimento da formulação, como por exemplo a solubilidade, a velocidade de dissolução e a forma das partículas. Para substâncias que oxidam com facilidade, por exemplo, deve-se adicionar o adjuvante farmacotécnico antioxidante. Assim como para os que sofrem degradação na presença de luz, a fotoproteção deve ser garantida para evitar a decomposição (VOGEL et al., 2020).

Os dados gerados nessa etapa permitem que se tome decisões a respeito da formulação de cada produto, indicando a forma farmacêutica mais apropriada, que pode ser líquida, sólida, semissólida ou gasosa, além do processo de fabricação mais adequado (AULTON; TAYLOR, 2016).

\subsection{Avaliação da estabilidade dos cosméticos}

A avaliação da estabilidade de um cosmético é fundamental, pois irá apontará o período de tempo durante o qual o produto mante suas propriedades físicas, químicas e microbiológicas dentro dos limites definidos e previamente estabelecidos (FRAZÃO, 2017). Amostras são avaliadas após serem submetidas a determinadas condições de temperatura, umidade e luminosidade, fatores que podem acelerar a velocidade de degradação dos componentes da formulação e, consequentemente, interferir na qualidade do produto (SIQUEIRA, 20I6; ROSÁRIO et al, 202I).

De acordo com a Agência Nacional de Vigilância Sanitária (ANVISA) a avaliação da estabilidade cosmética captura e fornece informações relacionadas ao grau relativo de estabilidade do produto nas diversas condições a que pode ser submetido, desde seu processamento e fabricação até o fim de sua eficácia (BRASIL, 2004).

Os testes são realizados quando há a criação de novos produtos, quando estes sofrem alterações significativas na formulação, para estimar o prazo de validade e gerar informações sobre sua confiabilidade e segurança. Eles também são usados para verificação de equipamentos ou processos de produção quando grandes mudanças ocorrerem nas matérias-primas e nos materiais de embalagem que ficam em contato direto 
com o produto (ALLEN JR; POPOVICH; ANSEL, 2013; FERREIRA; ZATTI, 2015; FERREIRA, 2016)

\subsection{Fatores que influenciam a estabilidade}

Segundo Rebello (2019) são variados os fatores que podem afetar a estabilidade de um produto, desde a matéria-prima escolhida e utilizada, condições ambientais e de transporte, até o seu processo de fabricação. Estes fatores podem ser classificados como extrínsecos ou intrínsecos.

Os fatores extrínsecos são fatores externos aos quais o produto fica exposto, como temperatura, luz, oxigênio, umidade, material de acondicionamento, microrganismos e vibração. (AULTON; TAYLOR, 2016, BRASIL, 2004). Produtos fotossensíveis, por exemplo, devem ser armazenados ao abrigo da luz, em frascos opacos ou escuros, pois a luz pode influenciar no processo de oxidação e decomposição (ROSÁRIO et al, 2021). Segundo Allen Jr, Popovich e Ansel (2013) a umidade afeta principalmente a forma de cosméticos sólidos, em que a aparência física do produto muda, tornando-o macio, pegajoso, ou mudando de peso ou volume, podendo ocorrer também a contaminação microbiana. $O$ transporte pode afetar a integridade das formulações com as vibrações que são geradas, podendo acarretar separação de fases de emulsões e alteração da viscosidade, por exemplo (BRASIL, 2008).

Os fatores intrínsecos, segundo Souza e Antunes (2018) são determinados por fatores inerentes à formulação e, sobretudo à interação entre os componentes da formulação com o material de acondicionamento.

\subsubsection{Parâmetros de avaliação da estabilidade}

Os parâmetros a serem avaliados no produto submetido ao teste de estabilidade dependem das características do produto em estudo e dos ingredientes que irão compor a formulação. De modo geral, os aspectos que geralmente são considerados como parâmetros de avaliação são: características organolépticas (cor, odor e sabor, quando aplicável), físicoquímicas e microbiológicas (FRANZEN et al, 2016).

Os parâmetros físico-químicos usualmente aplicados são a verificação do $\mathrm{pH}$, viscosidade, densidade e, em alguns casos, monitoramento dos componentes da formulação, efeito anticorrosivo e teor de ingredientes ativos. Em cada etapa de inspeção, 
deve-se verificar o cumprimento das boas práticas de fabricação e o sistema conservante utilizado na formulação (BRASIL, 2004; REBELLO, 2019).

$\mathrm{Na}$ observação dos parâmetros físicos é preciso manter as características físicas originais, como aparência, cor, cheiro e uniformidade. Nos parâmetros químicos, a integridade da estrutura química e o conteúdo dos ingredientes devem ser mantidos dentro dos limites especificados. Dentre os parâmetros microbiológicos, a carga microbiana do cosmético deve avaliada, assim como a atividade do sistema conservante utilizado (BRASIL, 2008; PATRICIO, 2020).

O parâmetro sensorial determinará os critérios de aceitação do produto pelo consumidor e as avaliações físico-químicas informarão conteúdos importantes para estudar as alterações na composição da fórmula (CALIXTO, 2020). Além desses aspectos, também é necessário considerar a manutenção das características do produto em termos de eficácia e segurança (BRASIL, 2012).

\subsubsection{Os testes de estabilidade e critérios para aprovação}

De acordo com Ferreira e Zatti (2015) e a ANVISA (BRASIL, 2004) os testes necessários para estudos de estabilidade dos cosméticos podem se dividir em centrifugação, estabilidade preliminar e acelerada, teste de prateleira, teste de transporte e distribuição e teste de compatibilidade entre formulação e material de acondicionamento.

Inicialmente recomenda-se submeter o produto ao teste de centrifugação, que deve permanecer estável, e qualquer indício de instabilidade acarretará em uma nova reformulação. Se aprovado nesse teste, o produto pode ser submetido aos testes de estabilidade posteriores (BRASIL, 2004).

A sequência dos estudos preliminares, acelerados e de prateleira têm como intuito avaliar a formulação em etapas, procurando indícios que auxiliam nas conclusões sobre a conservação do produto. Os testes devem ser realizados em condições que possam disponibilizar conteúdos sobre a perservação do produto no menor tempo possível (PAIVA, 20I6). Por esse motivo, as amostras devem ser guardadas em condições que possam acelerar possíveis alterações durante o prazo de validade (FRANZEN et al, 2016).

O estudo de estabilidade preliminar testa o produto no estágio inicial de desenvolvimento do produto. $\mathrm{O}$ uso de condições extremas de temperatura acelera possíveis reações e o aparecimento de sinais que devem ser observados e analisados. $\mathrm{O}$ 


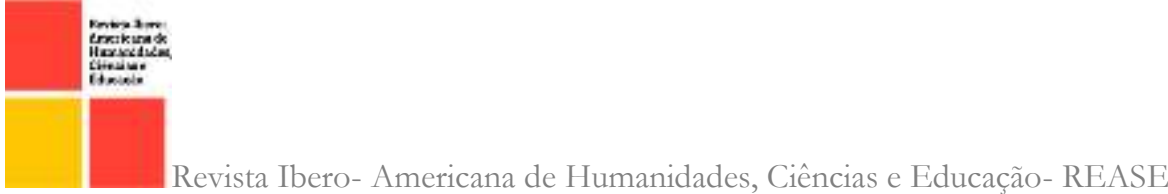

teste preliminar dura quinze dias e não tem o objetivo de estimar o prazo de validade do produto, mas de auxiliar na seleção da fórmula (BRASIL, 2004; CALIXTO, 2020).

O teste de estabilidade acelerada, ou exploratória, fornece informações para predizer as características do produto, o tempo de vida útil e a compatibilidade da formulação com o material de acondicionamento. É um estudo que dura cerca de noventa dias e pode estimar o prazo de validade do produto (BRASIL, 2004; SIQUEIRA, 2016).

O teste de prateleira, também conhecido como Shelf life (prazo de validade), verifica o limite de estabilidade do produto e comprova o prazo de validade estimado no teste de estabilidade acelerada (BRASIL, 2004; ROMERO, 2018).

Segundo o estudo apresentado por Souza e Antunes (2018), para a aprovação de um cosmético, o produto deve atender a todos os parâmetros estabelecidos pela agência reguladora, aos requisitos de qualidade estabelecidos pela indústria fabricante e às necessidades de desenvolvimento do produto apresentado na pré-formulação. As amostras que foram avaliadas devem permanecer inalteradas durante todo o ciclo de vida do produto. O período durante o qual um produto mantém suas características originais é denominado vida de prateleira, o que determina o prazo de validade (BRASIL, 2004; ROZENFELD, 2006; PAIVA, 2016).

Ao final dos estudos de estabilidade, é recomendado um relatório conclusivo, que inclui uma identificação do produto em análise, os materiais de embalagem utilizados no teste, as condições do estudo, os resultados, incluindo os prazos de conclusão, validade e responsabilidade da pessoa pelo estudo por meio de sua assinatura (PATRICIO, 2020).

\section{CONSIDERAÇÕES FINAIS}

Os produtos cosméticos são elaborados a partir de diversas matérias-primas e tipos de aplicação no organismo humano. A complexidade da formulação exige um criterioso processo de escolha dos componentes e do processo de fabricação, iniciado na fase de préformulação, etapa determinante na integridade final do produto. $O$ presente trabalho mostrou a importância da fase de desenvolvimento dos produtos cosméticos na estabilidade física, química e microbiológica, sendo possível observar, através dos autores citados, que os estudos de pré-formulação permitem identificar com antecedência possíveis problemas que podem comprometer a qualidade do produto acabado. Como a fase de elaboração da formulação, são tão importantes também, os testes de estabilidade, que 
auxiliam no monitoramento da estabilidade sensorial, física, química e microbiologia, gerando informações sobre a confiabilidade do produto, desde o desenvolvimento até o prazo de validade, garantindo, sobretudo, a eficácia e a segurança. O tema apresentado neste trabalho possibilita novas oportunidades de pesquisa, podendo ser estendido para as formas farmacêuticas específicas dos produtos cosméticos.

\section{REFERÊNCIAS BIBLIOGRÁFICAS}

ALLEN JR, Loyd V.; POPOVICH, Nicholas G.; ANSEL, Howard C. Formas Farmacêuticas e Sistemas de Liberação de Fármacos-9. Artmed Editora, 2013.

AUlTON, Michael E; TAYLOR, Kevin M. G. Aulton delineamento de formas farmacêuticas. [tradução Francisco Sandro Menezes]. - 4 ed. - Rio de Janeiro: Elsevier, 2016.

BRASIL, ANVISA. Agência Nacional de Vigilância Sanitária. Guia de Estabilidade de Produtos Cosméticos - I. ed. - Brasília: ANVISA, 2004. On-line. Disponível em: https://www.gov.br/anvisa/pt-br/centraisdeconteudo/publicacoes/cosmeticos/manuaise-guias/guia-de-estabilidade-de-cosmeticos.pdf/view. Acesso em 04 de Out de 202I.

BRASIL, ANVISA. Agência Nacional de Vigilância Sanitária. Guia de controle de 1083 qualidade de produtos cosméticos - 2. ed. - Revista Brasília: ANVISA, 2008.

BRASIL, ANVISA. Agência Nacional de Vigilância Sanitária. Guia de controle de qualidade de produtos cosméticos - 2. ed. - Revista Brasília: ANVISA, 2008.

BRASIL, ANVISA. Agência Nacional de Vigilância Sanitária. Guia para Avaliação de Segurança de Produtos Cosméticos - 2. ed. - Brasília: ANVISA, 2012. On-line. Disponível em: https://www.gov.br/anvisa/pt$\mathrm{br} /$ centraisdeconteudo/publicacoes/cosmeticos/manuais-e-guias/guia-para-avaliacao-deseguranca-de-produtos-cosmeticos.pdf/view. Acesso em o6 de Out de 2021.

BRASIL, ANVISA. Agência Nacional de Vigilância Sanitária. Resolução- RDC № o7, de ro de fevereiro de 2015. Disponível https://bvsms.saude.gov.br/bvs/saudelegis/anvisa/2015/rdc0007_10_02_2015.pdf. Acesso em or de Out de 202I.

CALIXTO, Lívia Salomão. Desenvolvimento de formulações cosméticas contendo ativos de origem natural: avaliação das propriedades físico-mecânicas, sensoriais e eficácia clínica. Tese de Doutorado. Faculdade de Ciências Farmacêuticas de Ribeirão Preto, Universidade de São Paulo, 202o.

CLEPF, Sabrina; MARTINELLI, Dante Pinheiro; CAMPOS, Patricia MBG Maia. Visão sistêmica no desenvolvimento de produtos cosméticos. Caderno Profissional de Marketing-UNIMEP, v. 3, n. 2, p. 36-47, 2015. 


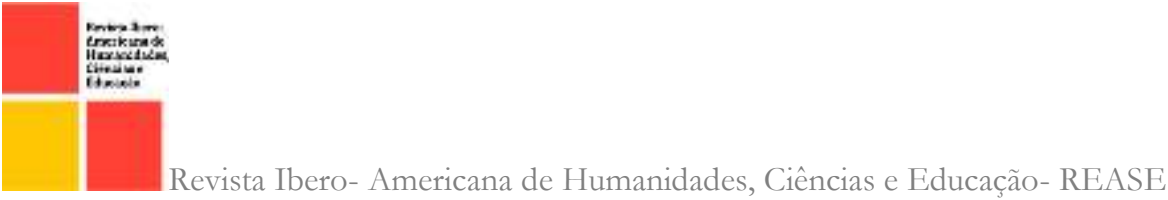

CORNÉliO, Melânia Lopes; ALMEIDA, Elaine Cristina Castro. Decifrando a composição dos cosméticos: riscos e benefícios. Uma visão do consumidor sobre o uso de produtos cosméticos. Brazilian Journal of Development, v. 6, n. 5, p. 30563-30575, 2020.

COSTA, Bruno Martins Galvão da et al. Acompanhamento do processo produtivo e controle de qualidade, na produção de géis e variados cosméticos da indústria salutaris. Relatório de Estágio Supervisionado apresentado à Universidade Federal Rural do SemiÁrido - UFERSA, Centro de Engenharias - CE para obtenção do título de Bacharel em Engenharia Química. MOSSORÓ - RN, 2018.

FERREIRA, Laura Victória Vieira. Nanotecnologia na formulação de cosméticos. Monografia realizada no âmbito da unidade de Estágio Curricular do Mestrado Integrado em Ciências Farmacêuticas, apresentada à Faculdade de Farmácia da Universidade de Coimbra. Coimbra, 2016.

FERREIRA, Raphael Marques; ZATTI, Ricardo Antonio. Protocolo para ensaios de estabilidade físico-química de fitocosméticos. ANAIS SIMPAC, v. 5, n. I, 2015.

FRANZEN, Jaqueline Maisa et al. Extrato hidroalcóolico de lactarius deliciosus incorporado à diferentes veículos cosméticos para testes de estabilidade. Revista Interdisciplinar de Estudos em Saúde, p. 137-I45, 2016.

FRAZÃO, Joana. Desenvolvimento de formulações cosméticas utilizando produtos apícolas e voláteis de cogumelos silvestres: determinação da estabilidade e toxicidade. Mestrado em cooperação com a Universidade de Salamanca. Mestrado em Farmácia e Quimica de Produtos Naturais, 2017.

FURTADO, Beatriz dos Anjos; SAMPAIO, Danilo de Oliveira. Cosméticos sustentáveis: quais fatores influenciam o consumo destes produtos?. International Journal of Business Marketing, v. 5, n. I, p. 36-54, 2020.

PAIVA, Maria Gabriela Broglio. Estudos de pré-formulação e desenvolvimento de cosméticos Linha Vivá. 20I6. $72 \mathrm{f}$. Trabalho de conclusão de curso (Farmácia-Bioquímica) Universidade Estadual Paulista, Faculdade de Ciências Farmacêuticas, 20r6. Disponível em: 〈http://hdl.handle.net/Ir449/203815>.

PATRICIO, Emanuella João. Avaliação da Estabilidade de um Produto Cosmético Formado por um Gel Hidrofílico. Engenharia Química-Tubarão, 2020.

REBELLO, Tereza. Guia de produtos cosméticos. I2 ${ }^{a}$ edição revista e ampliada. São Paulo: Editora Senac São Paulo, 20ı.

ROMERO, Valéria et al. Diferenças entre cosméticos orgânicos e naturais: literatura esclarecedora para prescritores. Surgical \& Cosmetic Dermatology, v. io, n. 3, p. I88-193, 2018. 
ROSÁRIO, Marcelino Santos do et al. Estudo de estabilidade de emulsão cosmética com potencial de creme hidratante para o tratamento da xerose cutânea utilizando o óleo de babaçu (Orbignya phalerata martius). Brazilian Journal of Development, v. 7, n. 3, p. 29552-29570, 2021.

ROZENFELD, Henrique et. al. Gestão de Desenvolvimento de Produtos: Uma Referência para a Melhoria do Processo. São Paulo: Editora Saraiva, 2006.

SILVA, Daniel Marcelino da. Análise e comparação das principais restrições químicas na formulação de cosméticos com base em legislações. 2017. $54 \mathrm{f}$. Trabalho de Conclusão de Curso (Graduação em Química) - Universidade Tecnológica Federal do Paraná, Curitiba, 2017.

SIQUEIRA, Jaqueline Cardoso de. Avaliação da estabilidade de uma emulsão cosmética cold cream contendo diferentes tipos de ceras. 20ı6. Monografia (Graduação em Química Industrial) - Universidade do Vale do Taquari - Univates, Lajeado, 29 nov. 2016. Disponível em: http://hdl.handle.net/ro737/r423. Acesso em 20 de Out 2021.

SOUZA, Ábila Baggi Reis; ANTUNES, Tamires Conceição Pires. Desenvolvimento de uma linha de aromas para uma indústria de cosméticos. Monografia (MBA Executivo em Gestão de Projetos) - Programa de PósGraduação, Centro Universitário SENAI CIMATEC, Salvador, 2018.

VOGEL, Eliane Maria et al. Avaliação da qualidade de cosméticos com ativo cafeína em bases galênicas gel e creme elaborado por farmácias magistrais na cidade de Campo Mourão-PR. Dissertação de Mestrado. Universidade Tecnológica Federal do Paraná, 2020. 